\title{
Ultra-compact plasmonic unidirectional wavelength multiplexer/ demultiplexer based on slot cavities
}

\author{
U. Aparna ${ }^{1} \cdot$ M. Sathish $\operatorname{Kumar}^{1}$ (i)
}

Received: 9 September 2021 / Accepted: 6 January 2022 / Published online: 17 January 2022

(c) The Author(s) 2022

\begin{abstract}
An ultra-compact plasmonic unidirectional wavelength multiplexer/demultiplexer based on slot cavities is proposed and numerically simulated. The structure consists of slot cavities which are etched on either sides of a metal-insulator-metal (MIM) bus waveguide. The cavities capture surface plasmon polariton (SPP) waves at their resonant wavelengths and unidirectionally couples them to the drop waveguide etched parallel to the bus. The structure basically relies on resonance and interference of SPP waves and its functioning is validated through finite element method (FEM) simulations. The simulation results show that the proposed structure functions as expected with a full width at half maximum (FWHM) bandwidth of less than $50 \mathrm{~nm}$, extinction ratio (ER) more than $10 \mathrm{~dB}$, and crosstalk (CT) of less than $-10 \mathrm{~dB}$ for the designed wavelengths. The proposed structure holds lot of potential to enhance the miniaturization of ultra-compact integrated photonic circuits for optical signal processing and other related applications.
\end{abstract}

Keywords Plasmonics $\cdot$ Plasmonic waveguide $\cdot$ Surface plasmon polariton $\cdot$ MIM devices $\cdot$ Multiplexer/demultiplexers

\section{Introduction}

The ability of surface plasmon polaritons (SPPs) to go beyond the conventional diffraction limit has made them potential candidates to realize nanoscale devices which can guide and manipulate light at subwavelength scales. This, in turn, can lead to construction of high density integrated optical circuits for transfer and processing of information [1-4]. With the rapid advances in nanoscale fabrication technology and easy availability of computational tools backed by affordable computational resources, researchers have been able to propose a variety of SPP based devices using a wide range of materials. Also, a few such devices with moderately complex structures have been fabricated [5-9]. Among these devices, those made of metal-insulator-metal (MIM) waveguides, which consists of a dielectric core and two metallic cladding layers, has drawn special attention due to their outstanding and significantly unique advantages. Such advantages include a strong subwavelength mode confinement

M. Sathish Kumar

sathish.kumar@manipal.edu

1 Department of Electronics and Communication Engineering, Manipal Institute of Technology, Manipal Academy of Higher Education, Manipal, Karnataka 576104, India in the dielectric core for a very wide range of wavelengths with an acceptable propagation length before the signal gets attenuated significantly due to metallic losses. [10,11]. Due to these advantages, research community has focused more on MIM waveguides and devices based on them such as filters, sensors, and lenses [12-15].

Multiplexing and demultiplexing being essential operations in communication and signal processing, plasmonic wavelength multiplexer (demultiplexer), which can collect different wavelengths and direct them into a single waveguide (and vice versa) has attracted a lot of interest recently. Such structures based on MIM waveguides have been found to be more interesting due to their advantages stated earlier. Due to their symmetry, simplicity, and ease of fabrication, optical resonators, which can select wavelengths, have been a key component in most of these structures [2]. Several types of disk, square and ring resonator were proposed in literature to design demultiplexers [10, 16-20]. Other approaches such as multimode interference to separate the wavelengths, focusing different wavelengths at different position in space, were also explored [8, 21-24].

In this paper, an ultra-compact wavelength demultiplexing structure based on plasmonic slot cavities is proposed and simulated. In comparison to other published work, our work differs in that we have proposed a structure wherein 
there is a drop waveguide and the dropped SPP can propagate in either of the two directions as per the designer's choice. The interplay between various design parameters of the structure were also investigated through simulations. The simulation was carried out using finite element method (FEM) and results so obtained demonstrate the functioning of the proposed structures as per the design objectives and specifications. By virtue of Helmholtz reciprocity theorem, the proposed demultiplexing structure can also function as a multiplexer if the input and output ports are swapped.

\section{Design and simulation}

Figure 1a shows the 2D schematic sketch and also illustrates the functioning of the proposed wavelength demultiplexer. SPP wave consisting of two wavelengths $\lambda_{1}$ and $\lambda_{2}$ propagates in the MIM bus waveguide at the centre. Two slot cavities are etched on either sides of the bus and are placed close enough to enable evanescent coupling of fields from the bus waveguide. The dimensions of these cavities are chosen such that they resonate for a specific wavelength. This ensures that they can drop specific wavelengths from the bus waveguide and evanescently couple them to the drop waveguides placed parallel on either sides of the bus.

It is assumed that the metal used in the proposed structure is gold with its complex relative permittivity calculated using Drude's model [25] while silica $\left(\mathrm{SiO}_{2}\right)$ with refractive index 1.2 is used as insulator. The wavelengths $\lambda_{1}$ and $\lambda_{2}$ were assumed to be $650 \mathrm{~nm}$ and $850 \mathrm{~nm}$, respectively. Width $w$ of all MIM waveguides, including that of the slot cavities, is considered as $100 \mathrm{~nm}$, which ensures that only the fundamental transverse magnetic $\left(\mathrm{TM}_{0}\right)$ mode is supported.

To enable evanescent coupling, the metal barrier thickness between the bus waveguide and the cavities, as well as between the cavities and the drop waveguides, is set as $30 \mathrm{~nm}$. The length $L$ of the cavity so that it resonates for a particular wavelength can be easily arrived at using [11],
$L=\frac{\left(m-\varphi_{r} / \pi\right) \lambda_{m}}{2 n_{\mathrm{eff}}}$

were $n_{\text {eff }}$ is the effective refractive index of the cavity experienced by the $m$ th resonating mode with wavelength $\lambda_{m}$ and $2 \varphi_{r}$ is the total phase shift accumulated due to reflection at the two facets per roundtrip. As shown in Fig. 1b where normalised output power $\left(P_{x}\right)$ vs cavity length $L_{1}$ for $650 \mathrm{~nm}$ is plotted, output power is maximum at a particular value of $L_{1}$, which is the resonating length. The same applies for $L_{2}$ for the $850 \mathrm{~nm}$ wavelength as shown in Fig. 1c. It may be observed from these figures that for resonance, $L_{1}=154 \mathrm{~nm}$ and $L_{2}=237 \mathrm{~nm}$.

\subsection{Parallel propagation in bus and drop waveguides}

In this subsection, design for direction of propagation of the wavelength dropped in the drop waveguide to be same as that of the bus waveguide is discussed. We call this as parallel propagation. If the direction of propagation in the bus and the drop waveguides are opposite to each other, we call that as antiparallel propagation. The latter is discussed in the next subsection.

Unless special care is taken, the dropped SPP wave will propagate in both the directions of the drop waveguide. To ensure unidirectional propagation, interference principle has to be exploited such that constructive interference of the dropped SPP wave happens in the desired direction of propagation while destructive interference happens in the opposite direction. The optical path length (OPL) of the fields propagating through the two cavities and combining in the drop waveguide will be the same in the direction in which the SPP wave is propagating in the bus. Needless to say, this will ensure constructive interference between the two fields from the cavities towards the direction of propagation of the SPP wave in the bus. However, to supress the SPP wave in (a)

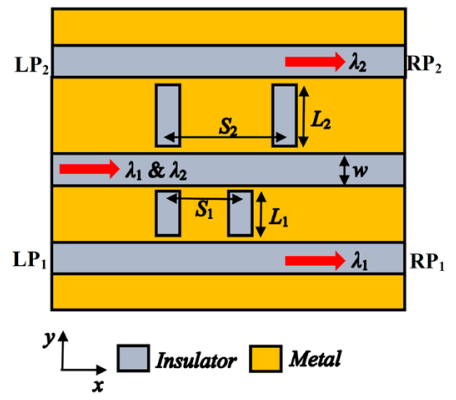

(b)

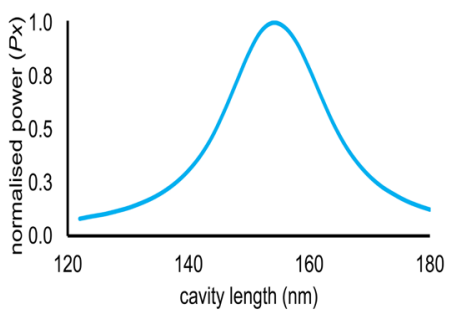

(c)

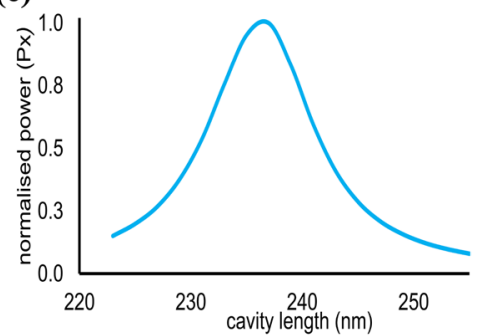

Fig. 1 Schematic sketch of the proposed demultiplexer is shown in (a). Dependence of normalised output power $\left(P_{x}\right)$ vs cavity length $\left(L_{1}\right)$ for $650 \mathrm{~nm}$ wavelength and cavity length $\left(L_{2}\right)$ for $850 \mathrm{~nm}$ wave- length is shown in (b) and (c) respectively. LP and RP stand for Left Port and Right Port 
the opposite direction, the difference in the OPL of the fields propagating through the cavities and combining in the other direction has to be such that destructive interference happens in the opposite direction. For this, the spacing $S$ between the cavities has to be arrived at from

$2 \beta S=(2 n+1) \pi$

were $\beta$ is the propagation constant of the SPP wave which can be obtained by solving the dispersion expression [1] and $n$ is an integer. With $n$ as 1 , the spacing $S=\frac{3 \pi}{2 \beta}$ provides a total phase accumulation of $1.5 \pi$ each for SPP wave between the cavities both in bus and drop waveguide. This will provide a relative phase difference of $3 \pi$ in the undesired direction between the SPP waves dropped by the two cavities which will result in destructive interference between them. This is expected to suppress the propagation in the other direction. A design of the structure as outlined in this paragraph results in a unidirectional demultiplexer which propagates the dropped wavelengths in the direction same as the direction of propagation of the SPP wave in the bus waveguide while suppressing it in the other direction.

Simulations were carried out using commercial FEM solver COMSOL Multiphysics. The $z$ component of the magnetic field intensity $\left(\left|H_{z}\right|^{2}\right)$ for the designed structure for $650 \mathrm{~nm}$ and $850 \mathrm{~nm}$ wavelengths are as shown in Fig. 2 with $S_{1}=314 \mathrm{~nm}$ and $S_{2}=427 \mathrm{~nm}$. From Fig. 2a, it can be observed that for the $650 \mathrm{~nm}$ wavelength, there is very little coupling of power into the top waveguide implying a very small crosstalk (CT). Figure $2 \mathrm{~b}$ demonstrates the same for $850 \mathrm{~nm}$. Overall, Fig. 2 demonstrates the successful separation of the two wavelengths. Our calculation shows that the CT for $650 \mathrm{~nm}$ is as small as $-19.09 \mathrm{~dB}$ while it was $-19.42 \mathrm{~dB}$ for $850 \mathrm{~nm}$. It is worth mentioning here that the CT was measured for $650 \mathrm{~nm}(850 \mathrm{~nm})$ as the ratio between power at $850 \mathrm{~nm}(650 \mathrm{~nm})$ and at $650 \mathrm{~nm}(850 \mathrm{~nm})$ computed at right port, $\mathrm{RP}_{1}$ (right port, $\mathrm{RP}_{2}$ ). We would like to add here that the coupling coefficient between the bus and the drop waveguide is not very large. However, this coupling coefficient can be improved through some structured optimization techniques which is not attempted in this work.

Though the above structure was designed for parallel propagation, Fig. 2 shows some propagation of field in the undesired direction. The reason for this is that though the fields add up out of phase in the undesired direction, the additional distance of $2 S$, which is twice the separation between the cavities, results in an extra attenuation of the field which propagates through the right cavity and interferes with the field from the left cavity. This results in an incomplete cancellation of fields due to their amplitude mismatch.

Extinction ratio (ER), which is a metric used to quantify the suppression of SPP wave in the undesired direction, is defined as the ratio of power at the desired port to the power at the undesired port [26]. In our above structure, the ER was found to be $6.3 \mathrm{~dB}$ for $650 \mathrm{~nm}$ and $7.66 \mathrm{~dB}$ for $850 \mathrm{~nm}$. As per the existing norms an ER $>10 \mathrm{~dB}$ is expected for practical applications [27]. This expectation motivated us to further investigate the above proposed structure's geometrical parameters.

Figure 3 shows the variation of ER with respect to the cavity length $L$ for both $650 \mathrm{~nm}$ as well as $850 \mathrm{~nm}$. It can clearly be seen that the ER can be increased by slightly
Fig. 2 Magnetic field intensity $\left(\left|H_{Z}\right|^{2}\right)$ distribution with $L_{1}=154 \mathrm{~nm}, L_{2}=237 \mathrm{~nm}$ for a $650 \mathrm{~nm}$ wavelength and $\mathbf{b}$ $850 \mathrm{~nm}$ wavelength, respectively.

Fig. 3 Plot of variation of ER and $\mathrm{CT}$ with respect to cavity length $L$ for $650 \mathrm{~nm}$ wavelength with $\mathrm{L}_{2}=237 \mathrm{~nm}$ in (a) and $850 \mathrm{~nm}$ wavelength with $L_{1}=154 \mathrm{~nm}$ in (b)
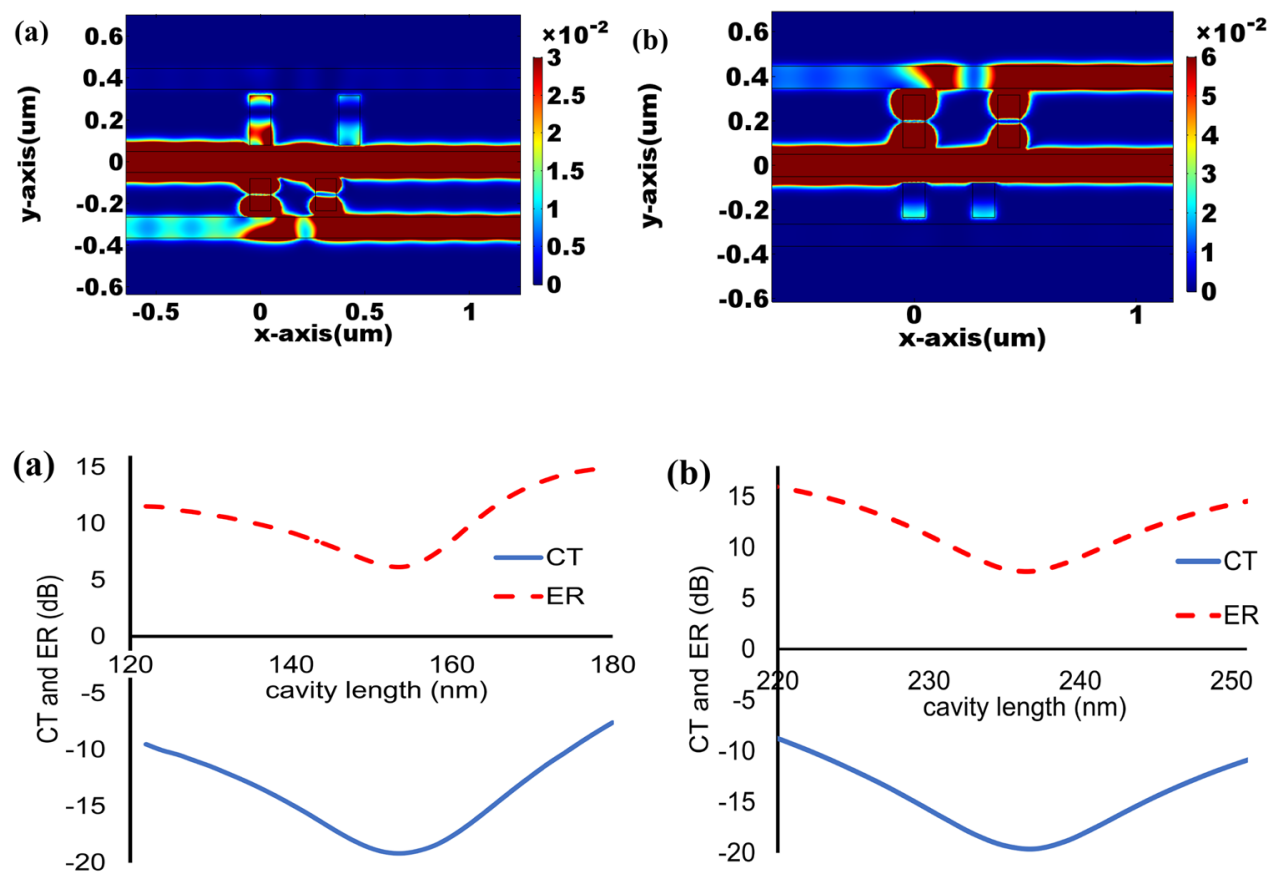
deviating $L$ from its optimum value possibly due to this facilitating better destructive interference. However, as can also be seen from this figure, CT also increases as $L$ deviates from its optimum value. The corresponding intensity plots are as shown in Fig. 4 for cavity lengths $L_{1}=165 \mathrm{~nm}$, and $L_{2}=243 \mathrm{~nm}$ for $650 \mathrm{~nm}$ and $850 \mathrm{~nm}$ wavelengths, respectively. These lengths, as can be seen from Fig. 3, can provide ER greater than $10 \mathrm{~dB}$.

Further, we also investigated the effect of slanting the cavities from their normal position. Intuitively, this direction of slant has to be towards the desired direction of propagation. Figure 5 shows the schematic of the structure with slant in the cavities as well as the variations in ER and CT as a function of angle of slant, $\theta$, with respect to the normal drawn to the bus axis. As can be seen earlier, ER as well as CT increases and it can be seen that at angle of
$13^{0}$ for both $650 \mathrm{~nm}$ as well as $850 \mathrm{~nm}$, the ER is around $10 \mathrm{~dB}$. Figure 6 shows the intensity plots with $L_{1}=154 \mathrm{~nm}$, $L_{2}=237 \mathrm{~nm}, \theta_{1}$ and $\theta_{2}=13^{\circ}$.

It may be noted that though increasing the angle would have improved the ER, it would have correspondingly made the CT more severe. The same argument applies to improving ER by deviating the cavity length from its optimum value. This clearly implies an underlying trade-off in the proposed structure.

To improve the coupling from the bus waveguide, number of cavities on either sides can be increased. However, it is worth to note that there is an upper limit on the number of cavities. As the number of cavities increase, the power from the bus drops rapidly and becomes negligibly small at some point. Increasing the cavities beyond this point will fetch no further increase in coupled power. A plot of ER, CT,
Fig. 4 Magnetic field intensity $\left(\left|H_{Z}\right|^{2}\right)$ distribution with $L_{1}=165 \mathrm{~nm}, L_{2}=243 \mathrm{~nm}$ for a $650 \mathrm{~nm}$ wavelength and $\mathbf{b}$ $850 \mathrm{~nm}$ wavelength, respectively
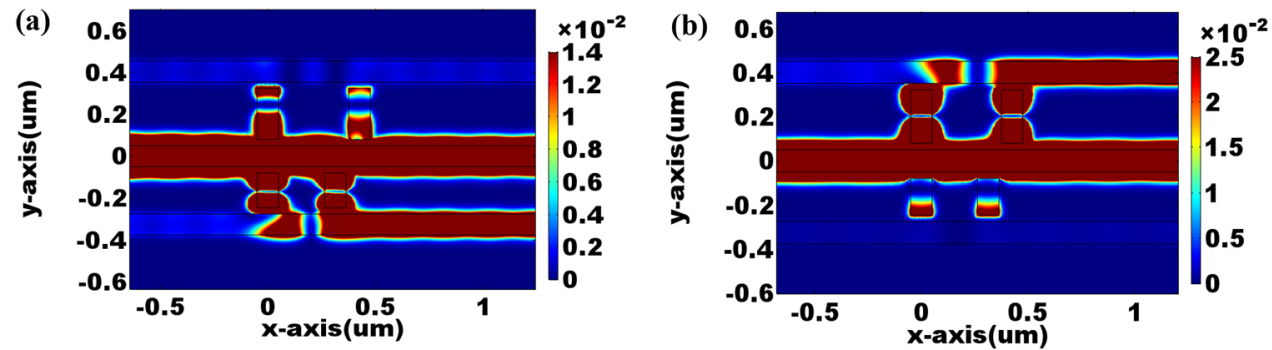

(a)

(a)
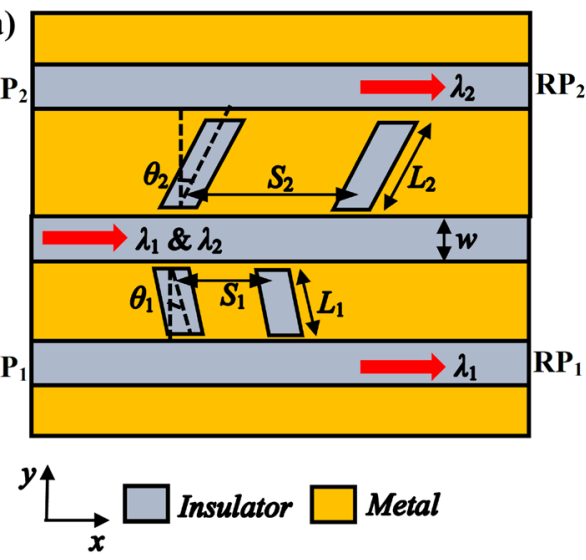

(b)

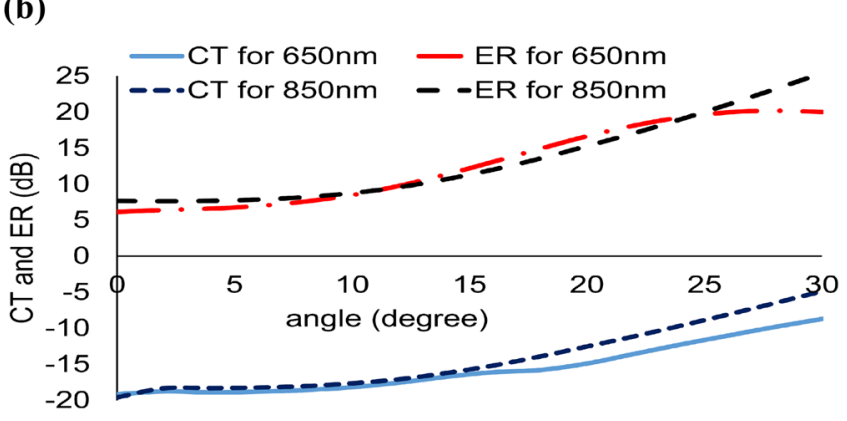

Fig. 5 a Modified schematic sketch of multiplexer/demultiplexer b plot of ER and CT vs angle with $L_{1}=154 \mathrm{~nm}, L_{2}=237 \mathrm{~nm}, \theta_{2}=13^{0}$ for $650 \mathrm{~nm}$ wavelength and $\theta_{1}=13^{\circ}$ for $850 \mathrm{~nm}$ wavelength

Fig. 6 Magnetic field intensity $\left(\left|H_{Z}\right|^{2}\right)$ distribution with $L_{1}=154 \mathrm{~nm}, L_{2}=237 \mathrm{~nm}$, $\theta_{1}$ and $\theta_{2}=13^{0}$ for $650 \mathrm{~nm}$ wavelength in (a) and $850 \mathrm{~nm}$ wavelength in (b) respectively
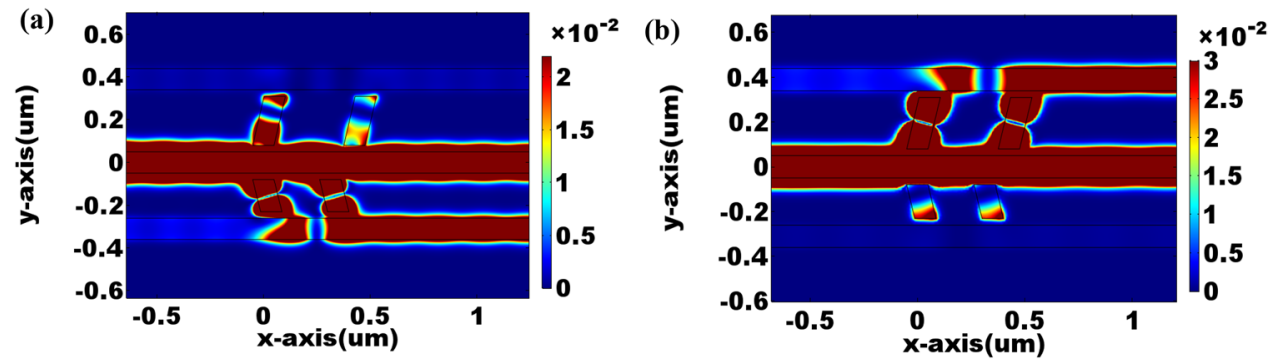
and normalized output power $\left(P_{x}\right)$ with respect to number of cavities with $L_{1}=154 \mathrm{~nm}, L_{2}=237 \mathrm{~nm}, \theta_{1}$ and $\theta_{2}=13^{\circ}$ for $650 \mathrm{~nm}$ and $850 \mathrm{~nm}$ wavelengths are shown in Fig. 7. It is clearly seen from this plot that there is an optimum value for the number of cavities. ER remains above $10 \mathrm{~dB}$ for two to nine cavities implying better destructive interference. Beyond 9 cavities, the ER drops because of less output power from the cavities. A similar behaviour can be observed with respect to CT also. Intensity distributions are shown in Fig. 8. The number of cavities is fixed at 5 on either sides of the bus. $L_{1}$ and $L_{2}$ are set as $154 \mathrm{~nm}$, and $237 \mathrm{~nm}$ with $\theta_{1}$ and $\theta_{2}=13^{\circ}$ for $650 \mathrm{~nm}$ and $850 \mathrm{~nm}$ wavelengths, respectively.

\subsection{Antiparallel propagation in bus and drop waveguides}

While the above discussion is for parallel propagation of coupled SPP, in the following, antiparallel propagation is discussed wherein the coupled SPP in the drop waveguide propagates in a direction opposite to the direction of propagation of the SPP in the bus. Slanting the cavities in the direction of desired propagation of the coupled SPP as it was done earlier does not work in this case. The reason being that to suppress coupled SPP in the undesired direction, the SPP waves from the cavities have to interfere destructively. However, since the phase acquired by the SPP waves meeting at the exit of the rightmost cavity will be the same, it is impossible to attain destructive interference in that direction. Consequently, a slight alteration in the orientation of the cavities is required here.

Figure 9 shows one possible structure with such alteration in the orientation of cavities. As can be seen, the rightmost cavity is perpendicular to the bus axis whereas the other defines an angle $\theta$. With the cavity slanted by an angle $\theta$, due to its increase in length compared to the straight cavity, loss in the slanted cavities will be relatively more. This hampers destructive interference of the dropped waves by the slanted-straight cavity pair in the undesired direction. To counter this, length of the straight cavity is slightly reduced. The angle $\theta$ is adjusted by trial and error so as to get the best possible ER and CT. Variation of CT and ER with respect to
Fig. 7 Plot of a ER and CT vs number of cavities $\mathbf{b}$ output power $\left(P_{x}\right)$ normalised with that of two cavities vs number of cavities with $L_{1}=154 \mathrm{~nm}$ and $L_{2}=237 \mathrm{~nm}, \theta_{1}$ and $\theta_{2}=13^{\circ}$ for $650 \mathrm{~nm}$ and $850 \mathrm{~nm}$ wavelength (a)

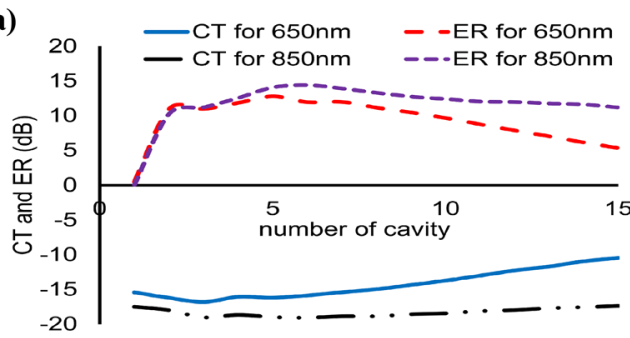

(b)
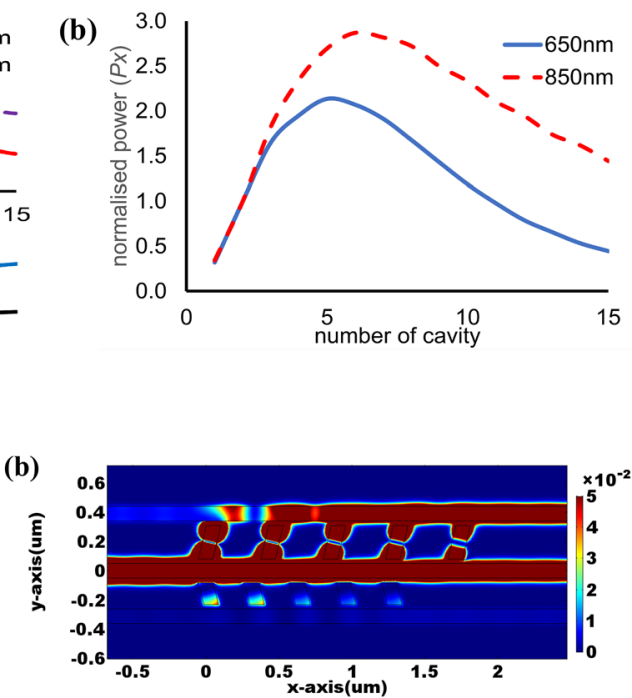

Fig. 8 Magnetic field intensity $\left(\left|H_{Z}\right|^{2}\right)$ distribution with 5 slant cavities on each side of bus with $L_{1}=154 \mathrm{~nm}, L_{2}=237 \mathrm{~nm}$, $\theta_{1}$ and $\theta_{2}=13^{\circ}$ for $650 \mathrm{~nm}$ wavelength in (a) and $850 \mathrm{~nm}$ wavelength in (b) respectively

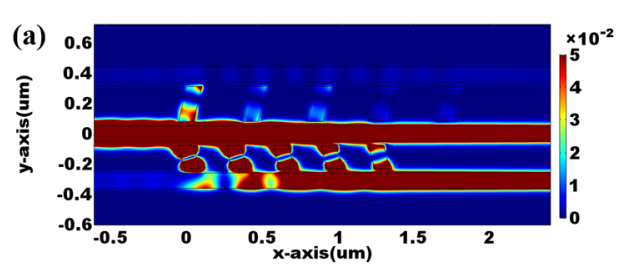

(b)

(a)

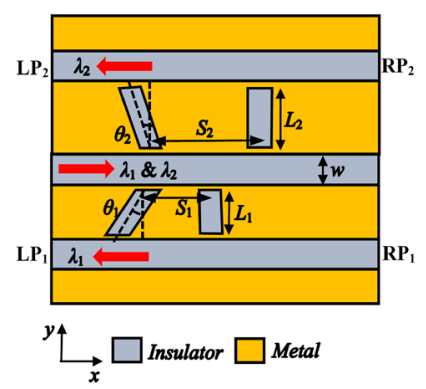

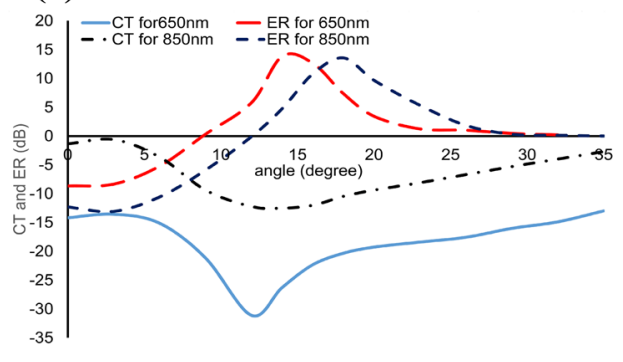

(c)

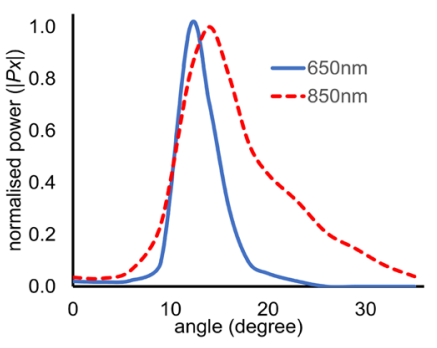

Fig. 9 a schematic sketch of Multiplexer/demultiplexer, plot of CT and ER vs $\theta$ in (b) and normalised output power ( $\left.\left|P_{x}\right|\right)$ vs $\theta$ in $(\mathbf{c})$ with $L_{1}=150 \mathrm{~nm}, L_{2}=228 \mathrm{~nm}, \theta_{2}=18^{\circ}$ for $650 \mathrm{~nm}$ and $\theta_{1}=16^{\circ}$ for $850 \mathrm{~nm}$ wavelength, respectively 
$\theta$ for the wavelengths $650 \mathrm{~nm}$ and $850 \mathrm{~nm}$ is also shown in this figure along with variation of normalized output power $\left|P_{x}\right|$ with respect to $\theta$. It can be seen that output power, ER, and $\mathrm{CT}$ take on their best possible values at different values of $\theta$ implying a trade-off when it comes to real life applications. Magnetic field intensity plots for the above structure for a few values of $\theta$ is shown in Fig. 10. The degree of left coupling as angle $\theta$ varies can be seen from this figure and a strong left coupling can be seen for an angle $\theta_{1}=16^{\circ}$ and $\theta_{2}=18^{\circ}$.

A plot of normalised power $\left(\left|P_{x}\right|\right)$ at both the desired right ports $\left(\mathrm{RP}_{1}\right.$ and $\left.\mathrm{RP}_{2}\right)$ as a function of wavelength is shown in Fig. 11a for parallel propagation as in the structure shown in Fig. 5a. The parameters considered for simulation are $L_{1}=154 \mathrm{~nm}, L_{2}=237 \mathrm{~nm}, \theta_{1}$ and $\theta_{2}=13^{\circ}$. Full Width at Half Maximum (FWHM) bandwidth of less than $40 \mathrm{~nm}$ for both $650 \mathrm{~nm}$ as well as $850 \mathrm{~nm}$ wavelength can be inferred from this plot. A similar plot for antiparallel propagation for the structure shown in Fig. 9a, wherein normalized power at left ports, $\mathrm{LP}_{1}$ and $\mathrm{LP}_{2}$ is plotted, is given in Fig. $11 \mathrm{~b}$. Simulation parameters considered are $L_{1}=150 \mathrm{~nm}, L_{2}=228 \mathrm{~nm}$, $\theta_{1}=16^{\circ}$ and $\theta_{2}=18^{\circ}$. It can be seen from this figure that the FWHM bandwidth for this case is less than $50 \mathrm{~nm}$ for both $650 \mathrm{~nm}$ and $850 \mathrm{~nm}$ wavelength.
We invite the attention of the reader to the fact that it is also possible to design the structure such that one of the wavelength will have parallel propagation whereas the other wavelength will have antiparallel propagation in the dropped waveguide. The schematic structure of such design is shown in Fig. 12a along with simulation results in Fig. 12b and c.

\subsection{Multiplexer and power splitter}

By virtue of Helmholtz reciprocity theorem, the above discussed demultiplexing structures can also act as multiplexers. For the case of the structure shown in Fig. $5 \mathrm{a}$, if $650 \mathrm{~nm}$ wavelength is fed as input through $\mathrm{RP}_{1}$ and simultaneously, $850 \mathrm{~nm}$ wavelength is fed as input through $\mathrm{RP}_{2}$, they will get coupled into the bus and propagate as a single SPP towards the left thus behaving as a multiplexer. Similarly, feeding $650 \mathrm{~nm}$ through $\mathrm{LP}_{1}$ and $850 \mathrm{~nm}$ through $\mathrm{LP}_{2}$, the structure shown in Fig. 9a will act as a multiplexer. Further, it is also possible to use these structures as power splitters by having cavities which resonate at the same wavelength on either side of the bus. Since these structures are etched on the sides of the bus waveguide, it is possible to have more than one functionality integrated along the same bus waveguide [24].

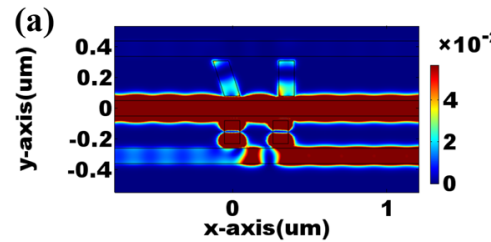

(d)

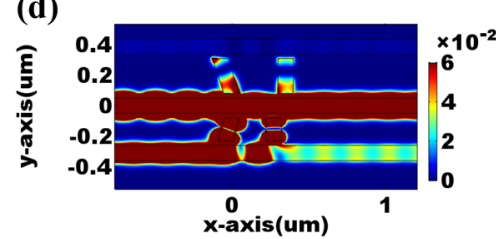

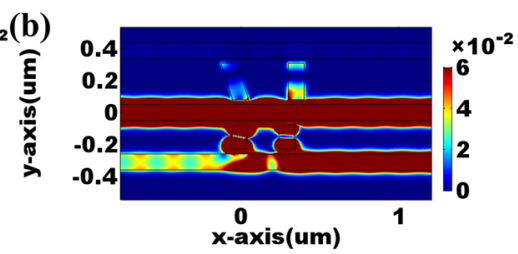

(e)

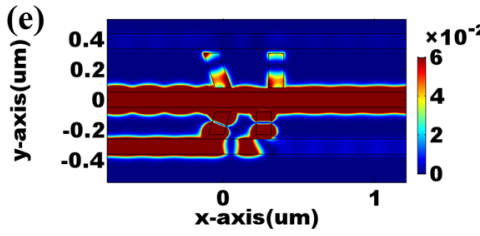

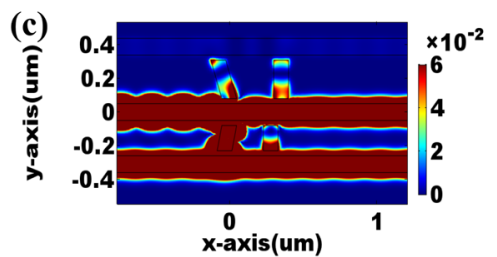

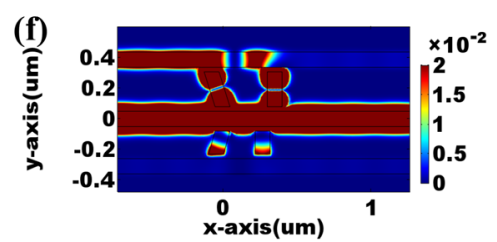

Fig. 10 Magnetic field intensity $\left(\left|H_{Z}\right|^{2}\right)$ distribution for $L_{1}=150 \mathrm{~nm}, L_{2}=228 \mathrm{~nm}, \theta_{2}=18^{\circ}$ with $\theta_{1}=0^{\circ}$ in $(\mathbf{a}), \theta_{1}=8^{\circ}$ in $(\mathbf{b}), \theta_{1}=12^{\circ}$ in $(\mathbf{c})$, $\theta_{1}=14^{\circ}$ in $(\mathbf{d}), \theta_{1}=16^{\circ}$ in (e) for $650 \mathrm{~nm}$ wavelength and $\theta_{1}=16^{\circ}$ with $\theta_{2}=18^{\circ}$ in (f) for $850 \mathrm{~nm}$ wavelength, respectively

Fig. 11 Plot of normalised power $\left(P_{x}\right)$ at desired output ports as a function of wavelength; a parallel propagation, and $\mathbf{b}$ antiparallel propagation
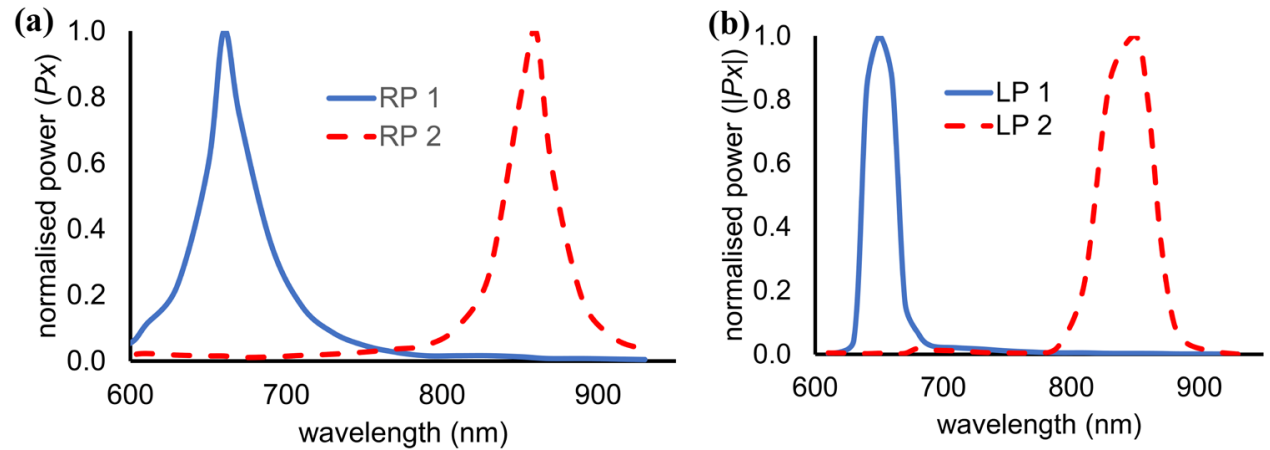
(a)

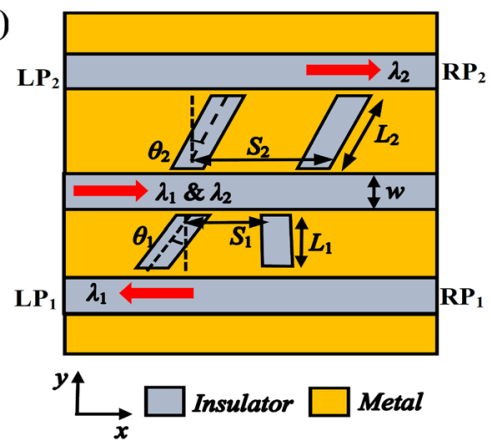

(b)

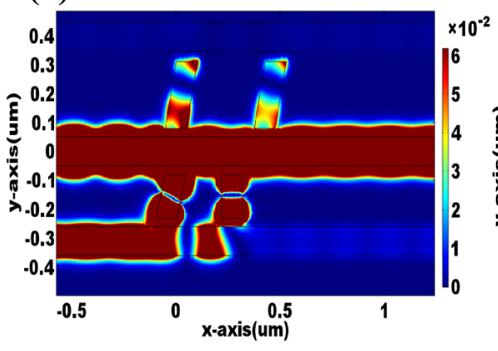

(c)

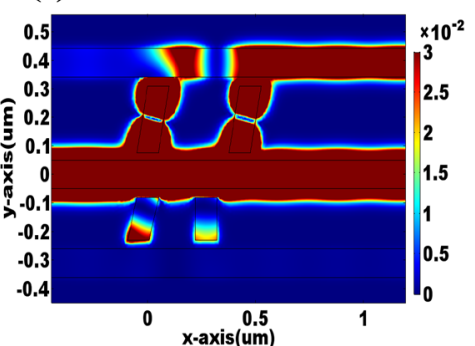

Fig. 12 a Schematic sketch of the proposed multiplexer/demultiplexer with dropped wavelengths propagating in opposite directions. Corresponding magnetic field intensity $\left(\left|H_{Z}\right|^{2}\right)$ distribution with

With this, it is felt that, the structures proposed in this paper is very versatile.

\section{Conclusion}

A two channel wavelength multiplexer/ demultiplexer is proposed and numerically simulated using FEM technique. Resonance and interference theory were used in the design which implies the wavelength chosen depends on the geometrical parameters of the structure. Simulation results shows a satisfactory ER of more than $10 \mathrm{~dB}$, cross talk of less than $-10 \mathrm{~dB}$ and a FWHM bandwidth of less than $50 \mathrm{~nm}$ for the chosen wavelengths of $650 \mathrm{~nm}$ and $850 \mathrm{~nm}$. Further, an ultra-small foot print of the structure makes it more suitable for future advanced photonic integrated circuits. As an additional advantage, several other signal processing structures can also be incorporated on the same bus one after the other, to provide a better integration feature.

Author contributions Theoretical ideas were developed by both the authors. Simulations were carried out by UA and MSK prepared the manuscript.

Funding Open access funding provided by Manipal Academy of Higher Education, Manipal. No funds, grants, or other support was received.

Availability of data and materials The datasets generated during and/or analysed during the current study are available from the corresponding author on reasonable request.

Code availability The code used during the current study are available from the corresponding author on reasonable request.
$L_{1}=150 \mathrm{~nm}, L_{2}=237 \mathrm{~nm}, \theta_{1}=16^{\circ}$ and $\theta_{2}=13^{\circ}$ for $650 \mathrm{~nm}$ wavelength in (b) and $850 \mathrm{~nm}$ wavelength in (c) respectively

\section{Declarations}

Conflict of interest The authors have no conflicts of interest to declare that are relevant to the content of this article.

Open Access This article is licensed under a Creative Commons Attribution 4.0 International License, which permits use, sharing, adaptation, distribution and reproduction in any medium or format, as long as you give appropriate credit to the original author(s) and the source, provide a link to the Creative Commons licence, and indicate if changes were made. The images or other third party material in this article are included in the article's Creative Commons licence, unless indicated otherwise in a credit line to the material. If material is not included in the article's Creative Commons licence and your intended use is not permitted by statutory regulation or exceeds the permitted use, you will need to obtain permission directly from the copyright holder. To view a copy of this licence, visit http://creativecommons.org/licenses/by/4.0/.

\section{References}

1. Maier, S.A.: Plasmonics: fundamentals and applications. Springer, Berlin (2007)

2. Lu, H., Liu, X., Gong, Y., Mao, D., Wang, L.: Enhancement of transmission efficiency of nanoplasmonic wavelength demultiplexer based on channel drop filters and reflection nanocavities. Opt. Express 19, 12885-12890 (2011)

3. Xie, Y.Y., He, C., Li, J.C., Song, T.T., Zhang, Z.D., Mao, Q.R.: Theoretical investigation of a plasmonic demultiplexer in MIM waveguide crossing with multiple side-coupled hexagonal resonators. IEEE Photonics J. 8, 1-12 (2016)

4. Khani, S., Danaie, M., Rezaei, P.: Design of a single-mode plasmonic bandpass filter using a hexagonal resonator coupled to graded-stub waveguides. Plasmonics 14, 53-62 (2019)

5. Yang, R., Lu, Z.: Subwavelength plasmonic waveguides and plasmonic materials. Int. J. Opt. 2012, 1-12 (2012)

6. Kamada, S., Okamoto, T., El-Zohary, S.E., Haraguchi, M.: Design optimization and fabrication of Mach-Zehnder interferometer based on MIM plasmonic waveguides. Opt. Express 24, 16224-16231 (2016)

7. Lee, J., Sung, S., Choi, J.H., Eom, S.C., Mortensen, N.A., Shin, J.H.: Ultra sub-wavelength surface plasmon confinement using air-gap, sub-wavelength ring resonator arrays. Sci. Rep. 6, 1-8 (2016) 
8. Zhang, S., Ji, W., Yin, R., Li, X., Gong, Z., Lv, L.: Full bandwidth wavelength division multiplexer/demultiplexer based on MMI. IEEE Photonics Technol. Lett. 30, 107-110 (2017)

9. Shao, L., Zheng, J.: Fabrication of plasmonic nanostructures by hole-mask colloidal lithography: recent development. Appl. Mater. Today 15, 6-17 (2019)

10. Lu, F., Wang, Z., Li, K., Xu, A.: A plasmonic triple-wavelength demultiplexing structure based on a MIM waveguide with sidecoupled nanodisk cavities. IEEE Trans. Nanotechnol. 12, 11851190 (2013)

11. Bavil, M.A., Gao, L., Sun, X.: A compact nanoplasmonics filter and intersection structure based on utilizing a slot cavity and a Fabry-Perot resonator. Plasmonics 8, 631-636 (2013)

12. Khani, S., Danaie, M., Rezaei, P.: Tunable single-mode bandpass filter based on metal-insulator-metal plasmonic coupled U-shaped cavities. IET Optoelectron. 3, 161-171 (2019)

13. Khajemiri, Z., Lee, D., Hamidi, S.M., Kim, D.S.: Rectangular plasmonic interferometer for high sensitive glycerol sensor. Sci. Rep. 9, 1-7 (2019)

14. Gao, Y., Liu, J., Guo, K., Gao, Y., Liu, S.: A side-illuminated plasmonic planar lens. Opt. Express 22, 699-706 (2014)

15. Shao, H., Wang, J., Liu, D., Hu, Z.D., Xia, X., Sang, T.: Plasmonic planar lens based on slanted nanoslit array. Plasmonics 12, 361-367 (2017)

16. Wang, G., Lu, H., Liu, X., Mao, D., Duan, L.: Tunable multichannel wavelength demultiplexer based on MIM plasmonic nanodisk resonators at telecommunication regime. Opt. Express 19, 3513-3518 (2011)

17. Zhang, Z., Yang, J., He, X., Han, Y., Zhang, J., Huang, J., Chen, D.: Plasmonic filter and demultiplexer based on square ring resonator. Appl. Sci. 8, 462 (2018)

18. Zafar, R., Chauhan, P., Salim, M., Singh, G.: Metallic slit-loaded ring resonator-based plasmonic demultiplexer with large crosstalk. Plasmonics 14, 1013-1017 (2019)

19. Nurmohammadi, T., Abbasian, K., Yadipour, R.: A proposal for a demultiplexer based on plasmonic metal-insulator-metal waveguide-coupled ring resonator operating in near-infrared spectrum. Optik 142, 550-556 (2017)

20. Azzazi, A., Swillam, M.A.: Nanoscale highly selective plasmonic quad wavelength demultiplexer based on a metal-insulator-metal. Opt. Commun. 344, 106-112 (2015)

21. Mu, J., Vázquez-Córdova, S.A., Sefunc, M.A., Yong, Y.S., GarcíaBlanco, S.M.: A low-loss and broadband mmi-based multi/demultiplexer in $\mathrm{Si}_{3} \mathrm{~N}_{4} / \mathrm{SiO}_{2}$ technology. J. Lightwave Technol. 34, 3603-3609 (2016)

22. Kumar, M.S., Piao, X., Koo, S., Yu, S., Park, N.: Out of plane mode conversion and manipulation of surface plasmon polariton Waves. Opt. Express 18, 8800-8805 (2010)

23. Wang, B., Wu, X., Zhang, Y.: Multiple-wavelength focusing and demultiplexing plasmonic lens based on asymmetric nanoslit arrays. Plasmonics 8, 1535-1541 (2013)

24. Aparna, U., Mruthyunjaya, H.S., Kumar, M.S.: Plasmonic wavelength demultiplexer with mode conversion capabilities. Plasmonics 13, 511-517 (2018)

25. Vial, A., Grimault, A.S., Macías, D., Barchiesi, D., de la Chapelle, M.L.: Improved analytical fit of gold dispersion: application to the modeling of extinction spectra with a finite-difference timedomain method. Phys. Rev. B 71, 085416 (2005)

26. Liu, D., Li, K., Lu, F., Ho, C.C., Xu, A.: Compact plasmonic unidirectional coupler with high performance based on an asymmetric slit-groove structure. J. Lightwave Technol. 32, 4837-4843 (2014)

27. Liu, D., Li, K., Ho, C.C., Xu, A., Li, H.: Efficiently unidirectional coupling of surface plasmons based on asymmetric cascaded nanoslits. IEEE Photonics Technol. Lett. 27, 1671-1674 (2015)

Publisher's Note Springer Nature remains neutral with regard to jurisdictional claims in published maps and institutional affiliations. 